\title{
Cellulose crushing: the experiment in the electromechanical transformer with a discrete secondary part
}

\author{
Alexander Lukyanov ${ }^{1 *}$, and Tatyana Onoiko ${ }^{1}$ \\ ${ }^{1}$ Don State Technical University, Department of automation of production processes, 344000 \\ Rostov-on-Don, Russia
}

\begin{abstract}
The electromechanical transformer with a discrete secondary part is developed. Researches on determination of duration of processing of suspension of cellulose on properties of cellulose fibers are conducted.
\end{abstract}

\section{Introduction}

For the purpose of confirmation of adequacy of a mathematical apparatus of the developed model and correctness of its assumptions experimental check of theoretical results of modeling was executed. At the same time the main task is experimental confirmation of the results received when modeling operation modes of the electromechanical transformer.

The solution of the following tasks was the purpose of carrying out pilot studies of the electromechanical transformer with a discrete secondary part:

1. Experimental check of adequacy of a mathematical model of electromagnetic processes in the working chamber of the device;

2. Justification and confirmation of efficiency of grind of cellulose hydrodynamic cavitation in the electromechanical transformer with a discrete secondary part.

In this case, should act as results of an experiment of assessment of electromagnetic processes: ultimate and active power, distribution of a discrete secondary part on the volume of the working chamber. Average length of fiber will act as results of assessment of efficiency of processing.

\section{Main part}

For a research the developed experimental sample of the electromechanical transformer from a discrete part was selected.

Production of a magnetic conductor of a magnet system can be made of different materials. However specific requirements are imposed to material of a magnetic conductor of a magnet system. The magnetic conductor should be made of the material having big magnetic permeability in order that the magnetic flux was created by smaller value of the magnetomotive force. As the inductor is subject to magnetic reversal that respectively leads

\footnotetext{
* Corresponding author: alexlukjanov1998@gmail.com
} 
to additional hysteresis losses and whirling currents, at material selection of a magnetic conductor it is necessary to be guided in addition to high-magnetic permeability, the increased unit resistance and minimum loss from magnetic reversal.

Electrotechnical steel are issued in the form of thin-sheet material. Thin electrotechnical sheet steel can be isotropic and anisotropic. Generally such provide to steel induction of 1.8-1.9 $\mathrm{T}$, and have rather high unit resistance and small hysteresis losses. At the same time the magnet system is gathered from single sheets blended) that allows to reduce influence of whirling currents.

Proceeding from features of operation of the projectible electromechanical transformer with a discrete secondary part, for such magnet systems the most suitable material is thinsheet $(0.35 \mathrm{~mm})$ electrotechnical steel 2214 with the content of silicon of $2.8-3.8 \%$. Silicon as doping impurity in steel, promotes increase in electrical resistance of steel that leads to decrease in eddy current loss, without increasing at the same time fragility of steel.

The electromechanical transformer in processing of cellulose assumes work with the increased heating of windings. Such conditions impose strict requirements to the materials which are a part of the device including, isolation of a winding should have heat stability not worse than category F (1550C).

The most suitable for production of coils of concentrated winding is the copper wire of round or rectangular section with the distinguished two-layer glass-fiber isolation impregnated with heatresistant silicon varnish.

The experimental sample of the electromechanical transformer with a discrete secondary part made according to results of researches has the following parameters.

1. Inductor parameters

- magnetic conductor material - electrotechnical steel of brand 2214;

- number of teeth - 10;

- number of phases -10 .

2. Parameters of a secondary part:

- quantity of elements - 400;

- an element form - a two-edged rod with a super cavitating profile;

- element length - $30 \mathrm{~mm}$;

- diameter of section of an element in the widest place $-3.6 \mathrm{~mm}$.

3. Inductor winding parameters:

- winding type - multiphase concentrated;

- wire section $-1.31 \mathrm{~mm}$.

The three-dimensional graphic model of the process of grind of suspension of the inductor of the electromechanical transformer made for implementation with a discrete secondary part is given in figure 1.

Main objective of these researches is assessment of an opportunity and efficiency of grind of cellulose by impact of hydrodynamic cavitation in the electromechanical transformer with a discrete secondary part. For achievement of a goal in the course of the researches it was required to evaluate the average length of fibers quantitatively. 


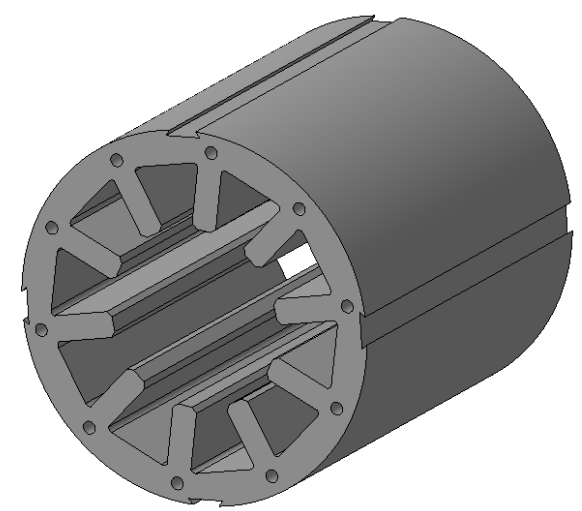

Fig. 1. Three-dimensional model of the inductor of the electromechanical transformer with a discrete secondary part.

For confirmation of impact of heavy traffic of big set of ferromagnetic elements on cellulose, a series of experiments was conducted. Experiments were made on an experimental sample of the device as which working chamber the glass from magnetically inert material which volume was 11 acted.

Before experience in the working chamber filled up a set of ferromagnetic elements. Pulp slurry was filled in in the reactor, turned on the electromechanical transformer with a discrete secondary part and the power unit regulating process of the movement of big set of ferromagnetic elements on an estimated trajectory. Through required time, installation disconnected, selected the processed material, again started installation, and continued raw materials processing.

The quality of process of grind of cellulose in the electromechanical transformer decided on a discrete secondary part by sampling of the suspension, which underwent processing in the device, each 20 min. For finding of optimum parameters of processing (time) when carrying out process of grind dependences of quality of grind of cellulose fiber on processing time were received. For avoiding of accidental errors in the course of the experiments and confirmation of stability of processing, a series of experiences was carried out.

The research of fractional structure of weight shows that it is possible to carry out grind due to fibrillation and splitting of fibers longwise at their insignificant shortening that has essential value for quality of the received weight. Processing by hydrodynamic cavitation of weight in the electromechanical transformer with a discrete secondary part promotes increase in speed of dehydration and decrease in its water retention. When using the electromechanical transformer with a discrete secondary part the amount of the fibers, which are dropping out in a deposit several times, decreases.

Results of tests of process of grind of cellulose fiber in the electromechanical transformer with a discrete secondary part are given in table 1.

Table 1. Grind of cellulose fiber

\begin{tabular}{|l|l|}
\hline Duration of grind, min. & Average is long fibers, $\mathrm{mm}$ \\
\hline 30 & 1,87 \\
\hline 60 & 1,72 \\
\hline 90 & 1,68 \\
\hline 135 & 1,4 \\
\hline
\end{tabular}


So as a result of our tests it is established that grind of suspension of cellulose allows to shorten length of cellulose fibers. The research of fractional structure of weight shows that it is possible to carry out grind due to fibrillation and splitting of fibers longwise at their insignificant shortening that has essential value for quality of the received weight. Cellulose suspension processing in the electromechanical transformer with a discrete secondary part promotes increase in speed of dehydration and decrease in its water retention. When using installation the amount of the fibers, which are dropping out in a deposit, decreases.

Authors express gratitude to scientists Minkin M.S. and Kuimov D.N. for the provided help in carrying out researches on studying of impact of hydrodynamic cavitation on cellulose suspension.

\section{Conclusion}

The conducted complex of pilot studies confirmed a hypothesis of efficiency of processing of suspension of cellulose with intensive impact of hydrodynamic cavitation. Cellulose suspension processing in the electromechanical transformer with a discrete secondary part promotes increase in speed of dehydration and decrease in its water retention. When using installation the amount of the fibers, which are dropping out in a deposit, decreases.

The reported study was funded by RFBR according to the research project № 18-29-18064.

\section{References}

1. D.N. Kuimov, A.V. Pavlenko, Lecture Notes in Mechanical Engineering, 4, 6 (2019)

2. D.N. Kuimov, A.V. Pavlenko, M.S. Minkin, MATEC Web of Conferences, 226, 6 (2018)

3. D.N. Kuimov, M.S. Minkin, Serbian journal of electrical engineering, 14, 3, 9 (2018)

4. V.N. Galushko T.V. Alferova, S.I. Bakhur, A.A. Alferov, the Messenger of the Gomel state technical university of P.O. Sukhy, 3, 11 (2014)

5. E.G. Andreyeva, A.A. Tatevosyan, I.A. Syomina, the Omsk scientific bulletin, 2, 5 (2013)

6. N.I. Neustroyev, the International scientific log alternative power engineering and ecology, 19, 6 (2014)

7. A.V. Shevkunova, New science: Problems and perspectives, 3, 4 (2016)

8. A.V. Kashuba, Works of the Rostov state transport university, 3, 12 (2016)

9. N.N. Klochkova, A.V. Obukhova, the Bulletin of the Samara state technical university. Series: Technical science, 2, 5 (2010)

10. D.N. Kuimov, M.S. Minkin, A.D. Lukyanov, Materials Science Forum, 870, 6 (2016) 